\title{
Automatic HDRI Generation of Dynamic Environments
}

Katrien Jacobs

VECG Lab, CS Department, UCL k.jacobs@cs.ucl.ac.uk

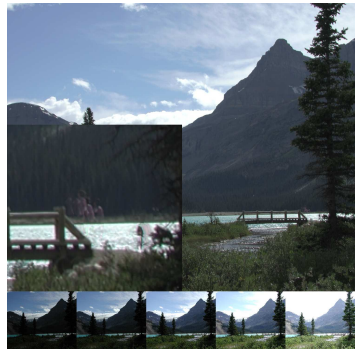

(a)

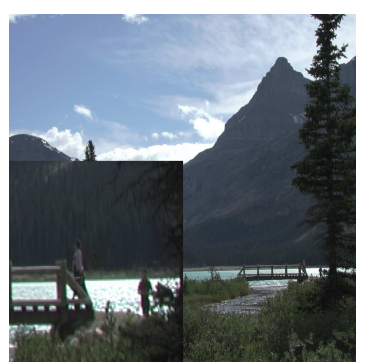

(b)
Greg Ward

Anyhere Software Ltd. gward@1mi.net

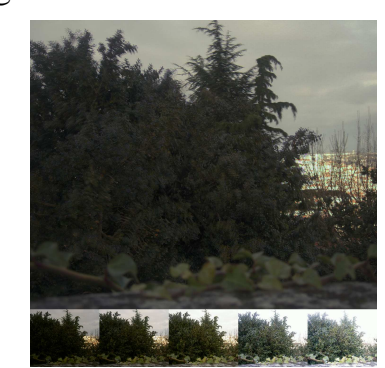

(c)
Celine Loscos

VECG Lab, CS Department, UCL

c.loscos@cs.ucl.ac.uk

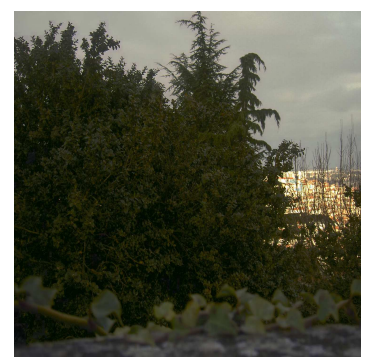

(d)

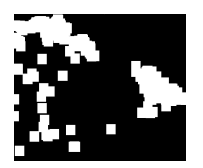

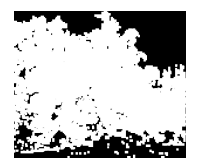

(e)

Figure 1: (a) The 5 LDRI segments show HCM (walking people) and the resulting HDRI (large image) shows an undesirable duplication of the human shapes (inset). (b) HDRI generation using the presented method, HCM is removed using a variance image, VI. (c) HDRI of a dynamic scene (larger image) with LCM (leaves). (d) HDRI after LCM removal using an uncertainty measure, UI. (e, top) VI segmentation. (e, bottom) UI segmentation.

\section{Introduction}

Conventional High Dynamic Range Image (HDRI) generation methods using multiple exposures [HDR Shop ][RASCAL ] require a static scene throughout the (Low Dynamic Range) image (LDRI) capturing. This hypothesis strongly limits the application field of HDRI generation. The presented system offers an automatic HDRI generation of dynamic environments with moving objects and camera misalignments.

Two types of motion are identified. High Contrast Movement (HCM) occurs when the dynamic object and background are significantly different; examples are moving people, cars, clouds, etc. Low Contrast Movement (LCM) is created when the dynamic object and the background are similar; examples are moving leaves, water rippling, etc. HCM and LCM result in blurring and duplication of information in the HDRI, see figure 1 (a)(c). The presented method detects and removes HCM and LCM using exposure invariant image statistics. The final HDRI is free from visible artefacts.

\section{Conventional HDRI generation}

Each pixel value in an HDRI is a weighted average of the matching pixel values in the $N$ LDRIs. The static scene prerequisite implies that these $N$ pixels correspond to the same scene point. The weights differ per pixel and ensure that saturated pixel values are discarded. Most methods align the $N$ LDRIs, usually using a feature dependent method, which is an unreliable method when used to align images captured with different exposures.

\section{HDRI generation of dynamic scenes}

Camera alignment: Prior to HCM and/or LCM detection and removal, $N-1$ Euclidean transformations (translation and rotation) are estimated to align the $N$ LDRIs using an exposure invariant method [Ward 2003]. Hereafter, the camera curve is used to transform the pixel values in the $N$ LDRIs to the radiance domain.

HCM detection: A Variance Image (VI) is constructed by calculating per pixel $j$ the weighted variance over the transformed radiance values in the $N$ LDRIs $L_{n}(j)$ :

$$
V I(j)=\frac{\sum_{n=1}^{N} w_{n j} I_{n j}^{2} \cdot \sum_{n=1}^{N} w_{n j}-\left(\sum_{n=1}^{N} w_{n j} I_{n j}\right)^{2}}{\left(\sum_{n=1}^{N} w_{n j} I_{n j}\right)^{2}}
$$

The weights $w_{n j}$ are the same weights as described in section 2 . A high VI entry indicates HCM. However, low contrast movement results in low VI entries, making VI unsuitable to detect LCM.

LCM detection: A suitable LCM measure is derived from entropy, which is insensitive to the level of contrast in a data set. The $N$ transformed LDRIs are divided into small segments $x_{n}$. Per segment the entropy $H\left(x_{n}\right)$ and the conditional entropy $H\left(x_{n} \mid x_{m}\right)$ between matching segments from different LDRIs are calculated using the histogram and joined histogram of the segments [Viola and Wells 1995]. An Uncertainty Image (UI) is created as follows:

$$
U I(x)=\sum_{n=1}^{N} H\left(x_{n}\right)+\sum_{n=1, n \neq m}^{N}\left(\sum_{m=1}^{N} H\left(x_{n} \mid x_{m}\right)\right)
$$

Again a weighted average can be used to discard information from saturated pixels. A high UI entry indicates a high entropic value (chaotic texture inside the segment) and a dissimilarity between the segments, see figure1 (e, bottom). However it is likely that regions with a high entropy but no motion are misclassified as LCM as well. This is not necessarily undesirable, as small camera misalignments are most likely to cause artefacts in those regions anyway.

HDRI generation: By applying a threshold, dilation and erosion on VI and UI, motion regions containing HCM and/or LCM are detected. Per motion region the best LDRI is chosen as that LDRI that contains the least saturation in that particular region. For all pixels outside a motion region, HDRI generation proceeds in the conventional manner. Pixels inside a motion region are substituted by the radiance value in the chosen LDRI.

\section{Results}

Figure 1 (a) shows 5 LDRIs segments (bottom) of a dynamic scene with HCM (walking people) and its derived HDRI using conventional HDRI generation methods (large image). A duplication effect is clearly visible (inset). After constructing VI, HCM is removed using the presented method resulting in (b). Another HDRI of a dynamic scene containing LCM is given in (c): the leaves of the bush are misaligned and create a significant amount of blurring. LCM removal results in (d). (e, top) illustrates that VI detects the HCM near the border of the bush but fails to detect the LCM inside the bush. The entropy measure detects LCM and HCM (e, bottom). Besides LCM, UI also detects HCM, however building UI is more time consuming than building VI.

\section{Conclusion}

A method is presented that enables HDRI generation using multiple exposures for dynamic scenes. After a translational and rotational camera alignment, the regions in the HDRI affected by motion are identified using statistical and exposure invariant measures. This information is used during the HDRI generation in order to correctly represent the dynamic objects in the final HDRI. The method can easily be integrated into existing HDRI software.

\section{References}

HDR SHOP. http://www.ict.usc.edu/graphics/HDRShop/.

RASCAL. http://www1.cs.columbia.edu/CAVE/tomoo/RRHomePage/rrhome.html.

Viola, P., AND WELLS, W. M. 1995. Alignment by maximization of mutual information. In Proceedings of the Fifth International Conference on Computer Vision. WARD, G. 2003. Fast, robust image registration for compositing high dynamic range photographs from hand-held exposures. Journal of Graphics Tools 8, 2, 17-30. 